\title{
DISCRETE-TIME QUEUEING NETWORKS WITH GEOMETRIC RELEASE PROBABILITIES
}

\author{
W. HENDERSON AND \\ P. G. TAYLOR, University of Adelaide
}

\begin{abstract}
This note is concerned with the continuing misconception that a discrete-time network of queues, with independent customer routing and the number of arrivals and services in a time interval following geometric and truncated geometric distributions respectively, has a product-form equilibrium distribution.

DISCRETE-TIME QUEUEING NETWORK; EXTENDED BERNOULLI QUEUES; GEOMETRIC RELEASE PROBABILITIES; PRODUCT FORM
\end{abstract}

AMS 1991 SUBJECT CLASSIFICATION: PRIMARY 60K25

SECONDARY 60K30; 90B22

\section{Introduction}

A number of recent contributions to the literature have been concerned with product-form equilibrium distributions in discrete-time queueing networks with batch movement of customers within each time interval. In particular we note the papers [1], [2], [6], [7] and [8]. Reference [2] introduced a general framework in which customers are released from queues, arrive to the network in batches, and are routed to other queues in a batch-dependent fashion, and yet a product-form equilibrium distribution holds. The model includes the product-form networks of [4], [5] and [8] as special cases. A further generalisation was introduced in [1] in particular to add some cases of state-dependent routing to the group of product-form networks defined in [2].

Both [1] and [2] discussed an example of 'extended Bernoulli queues', originally presented by Pujolle in [6] and now published in [7], in which the number of customers that arrive to the network in any time interval follows a geometric distribution and the number that are served at each queue obeys a truncated geometric distribution. Customers are routed around the network, after arrival or service, independently of one another. In [2] this example was shown to satisfy the criteria for a product-form equilibrium distribution, but only when the network consists of a single queue. In both [6] and [7] a network of queues was allowed and a product-form solution claimed. An example entitled 'Pujolle's network of extended Bernoulli queues' (Example 4.1.2) was discussed in [1] where a network product-form equilibrium distribution was obtained. In this example the geometric service and arrival probabilities of [6] were used. However, the network parameters are not exactly the same as in Pujolle's example because they do not correspond to independent routing of customers.

In this note we shall show that a network of extended Bernoulli queues, as originally defined in [6] and [7], does not possess product form and that, while Example 4.1.2 of [1] does possess product form, it is not a network of extended Bernoulli queues with independent customer routing. In Section 3 we present a counterexample which shows that the form of equilibrium distribution claimed in [6] and [7] does not hold. Although the example alone is

Received 9 August 1991; revision received 25 November 1991.

Postal address for both authors: Teletraffic Research Centre, Department of Applied Mathematics, The University of Adelaide, G.P.O. Box 498, Adelaide, SA 5001, Australia. 
sufficient to prove our contention, we discuss in Section 2 why the network does not satisfy the conditions presented in [1] and [2] and where the proof goes wrong in [7]. We use the latter approach because it is often beneficial to see the limits of applicability of a theory through an example which fails rather than only through examples which succeed.

We model the system described in [6] and [7] within the parameterisation and notation of [1], with the aim of showing that Theorem 3.4 of [1] does not apply to this example.

\section{The model}

Consider an open network of $N$ queues with customers of a single type arriving, circulating and leaving.

The probability that $g_{0}$ customers arrive to the network in a given time-slot is

$$
P\left(g_{0}\right)=\left(\frac{p}{1-p}\right)^{g_{0}}\left(\frac{1-2 p}{1-p}\right), \quad p<1 / 2
$$

and the probability that $g_{i}$, of the $n_{i}$ customers present at queue $i$, are served in a time-slot is

$$
R_{i}\left(g_{i}, n_{i}\right)=\frac{\left(\frac{p_{i}}{1-p_{i}}\right)^{g_{i}}}{\sum_{j=0}^{n_{i}}\left(\frac{p_{i}}{1-p_{i}}\right)^{j}}
$$

Customers are assumed to route among the queues in the network, independently of other routing customers, moving from queue $i$ to queue $j$ with probability $p_{i j}, 0 \leqq i, j \leqq N$. Queue 0 labels the outside.

The transition probabilities $q\left(\bar{g}, \bar{g}^{\prime}, \bar{n}-\bar{g}\right)$, of moving from state $\bar{n}$ to $\bar{n}-\bar{g}+\bar{g}^{\prime}$ via the batch $\bar{g}$ changing into $\bar{g}^{\prime}$, are then

$$
q\left(\bar{g}, \bar{g}^{\prime}, \bar{n}-\bar{g}\right)=\left[\prod_{i=1}^{N} R_{i}\left(g_{i}, n_{i}\right)\right] \times\left[\sum_{g_{0}=0}^{\infty} \sum_{g_{0}^{\prime}=0}^{\infty} P\left(g_{0}\right) \sum_{g_{i j} \in S} \prod_{i=0}^{N}\left(\begin{array}{c}
g_{i} \\
g_{i 0^{*}} \cdots g_{i N}
\end{array}\right) \prod_{j=0}^{N} p_{i j}^{g_{i j}}\right]
$$

where

$$
S=\left\{g_{i j} \geqq 0: g_{i j}=0 \text { if } p_{i j}=0, g_{00}=0, \sum_{j=0}^{N} g_{i j}=g_{i} \text { and } \sum_{i=0}^{N} g_{i j}=g_{j}^{\prime}\right\}
$$

as defined in Equation (3.18) of [1]. We may rewrite (1) as

$$
\begin{aligned}
q\left(\bar{g}, \bar{g}^{\prime}, \bar{n}-\bar{g}\right) & =\left[\prod_{i=1}^{N} R_{i}\left(g_{i}, n_{i}\right) g_{i} !\right] \times\left[\sum_{g_{0}=0}^{\infty} \sum_{g^{\prime}=0}^{\infty} P\left(g_{0}\right) \sum_{g_{i j} \in S} \prod_{i=0}^{N} \prod_{j=0}^{N} \frac{p_{i j}^{g_{i j}}}{g_{i j} !}\right] \\
& =\left[\prod_{i=1}^{N} R_{i}\left(g_{i}, n_{i}\right) g_{i} !\right] \times \lambda\left(\bar{g}, \bar{g}^{\prime}\right) .
\end{aligned}
$$

The batch transition rate $\lambda\left(\bar{g}, \bar{g}^{\prime}\right)$ is defined in [1], where it is shown in Appendix A.3 that the solution of

$$
\sum_{\bar{g}} y(\bar{g}) \lambda\left(\bar{g}, \bar{g}^{\prime}\right)=\sum_{\bar{g}} y\left(\bar{g}^{\prime}\right) \lambda\left(\bar{g}^{\prime}, \bar{g}\right)
$$

is given by

$$
y(\bar{g})=\prod_{i=1}^{N}\left(\frac{p \gamma_{i}}{1-p}\right)^{g_{i}}
$$

with $\gamma_{i}$ satisfying $\gamma_{j}=\gamma_{0} p_{0 j}+\sum_{i=1}^{N} \gamma_{i} p_{i j}$ and $\gamma_{0}=1$. 
Consequently the $\pi_{y}$ part of the stationary distribution (cf. Theorem 3.4 , p. 164, of [1]) is

$$
\pi_{y}(\bar{n})=\prod_{i=1}^{N}\left(\frac{p \gamma_{i}}{1-p}\right)^{n_{i}}
$$

as given on p. 177 of [1].

The factor $\prod_{i=1}^{N} R_{i}\left(g_{i}, n_{i}\right)$, in the right-hand side of (2) can be written in the form $f(\bar{g}, \bar{n})=\psi(\bar{n}-\bar{g}) / \phi(\bar{n})$. However, when the factor $\prod_{i=1}^{N} g_{i} !$ is included, the bracketed term on the right-hand side of (2) cannot be written in this form. Consequently Theorem 3.4 of [1] cannot be applied.

The alternative approach is to include $\prod_{i=1}^{N} g_{i}$ ! in the routing part of the decomposition, i.e. in $\lambda\left(\bar{g}, \bar{g}^{\prime}\right)$. In this case the solution to (3) becomes

$$
y(\bar{g})=\prod_{i=1}^{N} \frac{\left(\frac{p \gamma_{i}}{1-p}\right)^{g_{i}}}{g_{i} !} .
$$

The $q_{y}$-process, as defined by Equation (3.7) of [1], needs an invariant measure satisfying

$$
\frac{\pi_{y}\left(\bar{n}^{\prime}\right)}{\pi_{y}(\bar{n})}=\frac{y\left(\bar{g}^{\prime}\right)}{y(\bar{g})}
$$

for the process to be strongly reversible. When $y(\bar{g})$ has the geometric form (4) the geometric equilibrium distribution (5) follows easily from (6). However, with the inclusion of $\prod_{i=1}^{N}\left(1 / g_{i} !\right)$, (6) cannot be satisfied except in very special cases. Consequently Theorem 3.4 of [1] is again inapplicable.

In Appendix A3 of [1] the authors showed that a network with transition probabilities given by (2), without the $\prod_{i=1}^{N} g_{i} !$, has a product-form equilibrium distribution. As we have shown above, this result is perfectly correct but it does not apply to a network of extended Bernoulli queues as originally defined in [6] and [7].

Now let us turn to the proof in [6] and [7] in which the author studies a network of the same form as that described above. He also studies a closed version of the network. The equilibrium distribution given in [7] is for time points when customers are en route in the network. A discussion of the relationship between equilibrium distributions at these time points and at time points before customers depart is given in [3] where such a distribution is referred to as the equilibrium distribution for base-states. The process which evolves on base-states is not a Markov chain, and can only be studied by incorporating information on the customers en route.

The method of proof given on p. 242 of [7] is to balance forward and reverse time fluxes for a transition between a base-state $\bar{m}$ with customers en route from queue $j$ to queue $i$ given by $g_{j i}$ to a base-state $\bar{m}^{\prime}=\bar{m}+\left[\sum_{j=0}^{N} g_{j i}\right] \bar{e}_{i}-\sum_{j=0}^{N} g_{i j} \bar{e}_{j}$ with $\bar{e}_{i}$ denoting the $i$ th unit vector. Here the difference between $\bar{m}$ and $\bar{m}^{\prime}$ implicitly involves the assumption that all customers intermediately reside in queue $i$. This is verified for all $i$, thus showing that forward versus reverse time balance holds for all transitions in which customers intermediately reside in any one queue. However, these are not the only transitions that can occur, since customers may reside intermediately at multiple queues. The proof in [6] and [7] does not allow for such transitions.

\section{Counterexample}

In this section we provide a counterexample which shows that a network of queues with geometric service and independent customer routing does not have the product-form distribution claimed in [6] and [7]. For simplicity we consider a closed network, specifically the simple two-node network of Figure 1 with routing probabilities as given. 


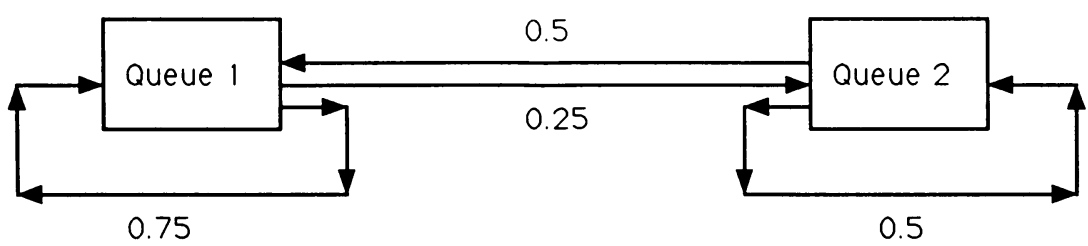

Figure 1

Let

$$
p_{i}= \begin{cases}\frac{1}{2}, & i=1 \\ \frac{1}{3}, & i=2\end{cases}
$$

then

$$
\frac{p_{i}}{1-p_{i}}= \begin{cases}1, & i=1 \\ \frac{1}{2}, & i=2\end{cases}
$$

The routing probabilities given in Figure 1 lead to $\gamma_{1}=2, \gamma_{2}=1$. Assume that there are two customers in the network.

The invariant measure proposed in Example 4.1.2 of [1] is $\pi\left(n_{1}, n_{2}\right)=\prod_{i=1}^{2}\left[R_{i}\left(n_{i}, n_{i}\right)\right]^{-1} \gamma_{i}^{n_{i}}$. Therefore, if this example were the same as that of [6] and [7],

$$
\pi(2,0)=12, \quad \pi(1,1)=12, \quad \pi(0,2)=7
$$

should satisfy the global balance equations.

For state $(2,0)$ the global balance equations are

$$
\begin{aligned}
\pi(2,0)= & \pi(2,0)\left[\frac{1}{3} \times\left(\frac{3}{4}\right)^{2}+\frac{1}{3} \times \frac{3}{4}+\frac{1}{3}\right] \\
& +\pi(1,1)\left[\frac{1}{2} \times \frac{1}{3} \times \frac{3}{4} \times \frac{1}{2}+\frac{1}{2} \times \frac{1}{3} \times \frac{1}{2}\right]+\pi(0,2)\left[\frac{1}{7} \times \frac{1}{2} \times \frac{1}{2}\right] .
\end{aligned}
$$

Substitution of (7) into the right-hand side of (8) gives 11.25 and not 12 , thus verifying that Example 4.1.2 is not a network of extended Bernoulli queues with independent customer routing.

On the other hand in [6] and [7] the equilibrium distribution

$$
\pi_{b}\left(m_{1}, m_{2}\right)=B \prod_{i=1}^{2}\left[\frac{\left(1-p_{i}\right) \gamma_{i}}{p_{i}}\right]^{m_{i}}
$$

is proposed for time points when customers are en route. The possible states at these time points are $(2,0),(1,1),(0,2),(1,0),(0,1)$ and $(0,0)$. For the first three of these base-states the equilibrium distribution given by $(9)$ is of the form $\pi_{b}(2,0)=\pi_{b}(1,1)=\pi_{b}(0,2)=4 B$. These particular $\pi_{b}\left(m_{1}, m_{2}\right)$ are related to the $\pi\left(n_{1}, n_{2}\right)$ by

$$
\begin{gathered}
\pi_{b}(2,0)=\pi(2,0) P(\text { nothing released from queue } 1)=\pi(2,0) / 3, \\
\pi_{b}(1,1)=\pi(1,1) P(\text { nothing released from queues } 1 \text { and } 2)=\pi(1,1) / 3, \\
\pi_{b}(0,2)=\pi(0,2) P(\text { nothing released from queue } 2)=4 \pi(0,2) / 7
\end{gathered}
$$

Therefore, assuming the equilibrium distribution (9) is correct, an invariant measure for 
time points at which all customers are present at queues has the ratio $12: 12: 7$ for states $(2,0),(1,1)$ and $(0,2)$ i.e. precisely the same measure as proposed in $(7)$. However, we have already shown that this is not the invariant measure for the network. Therefore the equilibrium distribution claimed in [6] and [7] is also incorrect.

\section{Conclusion}

We have shown that the product-form equilibrium distribution claimed in [6] and [7], for a network of queues with batch geometric services and independent routing of customers, does not hold. In addition we have clarified that Example 4.1.2 in [1], though entitled 'Pujolle's network of extended Bernoulli queues' does not, in fact, discuss the same model as [6] and [7].

\section{References}

[1] Boucherie, R. J. AND VAN DiJk, N. M. (1991) Product forms for queueing networks with state dependent multiple job transitions. Adv. Appl. Prob. 23, 152-187.

[2] Henderson, W. AND TAYLOR, P. G. (1990) Product form in networks of queues with batch arrivals and batch services. QUESTA 6, 71-88.

[3] Henderson, W. AND TAYLOR, P. G. (1991) Some new results on queueing networks with batch movements. J. Appl. Prob. 28, 409-421.

[4] JACKSON, J. (1957) Networks of waiting lines. Operat. Res. 5, 518-521.

[5] JACKsON, J. (1963) Jobshop-like queueing systems. Management Sci. 10, 131-142.

[6] Pujolle, G. (1988) Multiclass discrete time queueing systems with a product form solution. Preprint, Laboratoire MASI, Université Pierre et Marie Curie, Paris.

[7] Pujolle, G. (1991) Discrete-time queueing systems for data networks performance evaluation. In Queueing Performance and Control in ATM (ITC-13), ed. J. W. Cohen and C. D. Pack. Elsevier Science Publishers (North-Holland), Amsterdam.

[8] Walrand, J. (1983) A discrete-time queueing network. J. Appl. Prob. 20, 903-909. 